\title{
O TRATAMENTO RESTAURADOR ATRAUMÁTICO E A PANDEMIA DO SARS-COV-2
}

\author{
The atraumatic restorative treatment and the SARS-CoV-2 pandemic
}

\section{Larissa Maia Sousa Reis ${ }^{1}$ Júlia Alves de Paula ${ }^{1}$ Gabriel do Amaral Silva ${ }^{1}$ Antônio Sérgio Netto Valladão ${ }^{1}$}

${ }^{1}$ Centro Universitário de Valença (UNIFAA) Valença $(R J)$

Autor correspondente:

Antônio Sérgio Netto Valladão E-mail: antonio.valladao@faa.edu.br

\section{Como citar este artigo:}

REIS, L. M. S.; PAULA, J.A.; SILVA, G.A.; VALLADÃO, A.S.N. O tratamento restaurador atraumático e a pandemia do SARS-COV-2. Revista Saber Digital, v. 14, n. 3, p. 83-93, 2021.

Data de Submissão: 28/11/21

Data de aprovação: 16/12/21

Data de publicação: 21/12/21

\section{(c) (1) (8)}

Esta obra está licenciada com uma licença

http://creativecommons.org/licenses/by-nc/4.0/

\section{RESUMO}

Objetivo: Avaliar as indicações do tratamento restaurador atraumático, durante a pandemia do SARS-CoV-2. Materiais e Métodos: As etapas desta revisão integrativa foram delineadas e orientaram as fases da pesquisa, tendo início com o desenvolvimento da pergunta norteadora "Por que o TRA foi indicado na pandemia do SARS Cov-2?". As buscas foram realizadas nas bases de dados PubMed, Lilacs e Scielo e incluiram artigos originais e revisões bibliográficas, publicadas entre 2016 e 2021. Resultados: A partir de nove artigos selecionados, os resultados obtidos através da utilização do instrumento adaptado de Ursi mostraram que o TRA foi indicado para a redução do aerossol, proteção de pacientes e equipe de atendimento, prevenção da transmissão, conforto do paciente e como medida especial de segurança. Conclusões: Considerando o contexto da pandemia do SARS-CoV-2 e as indicações do TRA, esta revisão integrativa mostra que o TRA está indicado para o tratamento de pacientes, sobretudo por evitar a propagação do aerossol.

Palavras-chave: Tratamento Restaurador Atraumático, SARS-CoV2, COVID-19; Pandemia.

\section{ABSTRACT}

Objective: To evaluate the indications of the atraumatic restorative treatment during the SARS-Cov-2 pandemic. Methods: The steps of this integrative review were outlined and guided the research, starting with the development of the guiding question "Why ART was indicated in the SARS-CoV-2 pandemic?". Searches were performed in the PubMed, Lilacs and Scielo databases and included original articles and bibliographic reviews, published between 2016 and 2021 . Results: From nine selected articles, the obtained results using the instrument adapted from Ursi showed that the ART was indicated for the reduction of aerosol, protection of patients and dental staff, prevention of transmission, patient comfort and as a special safety measure. Conclusions: Considering the context of the SARS-CoV-2 pandemic and the indications of the ART, this integrative review shows that the ART is indicated for the treatment of patients, mainly because it avoids the spread of the aerosol.

Keywords: Atraumatic RestorativeTtreatment, SARS-CoV-2, COVID-19, Pandemic. 


\section{INTRODUÇÃO}

A doença COVID-19 causada pelo novo coronavírus (SARS-CoV-2) mostrou um impacto, sem precedentes, na população mundial e expôs os serviços de saúde a grandes desafios (ALI; RAJA, 2020), exigindo agilidade nas pesquisas médicas e odontológicas em face à urgência da necessidade de resultados (KANDEEL; AL-NAZAWI, 2020).

Do ponto de vista odontológico, a grande preocupação relaciona-se ao fato do SARS-CoV-2 ter sido encontrado em secreções nasofaríngeas e na saliva. Desta forma, além do contato direto com indivíduos infectados e objetos inanimados, a propagação da infecção ocorre através de gotículas expelidas durante a respiração. Tais gotículas, relacionadas ou não à geração de aerossol, associam-se ao alto risco de exposição dos cirurgiões-dentistas, uma vez que podem conter SARS-CoV-2 e através dos olhos, nariz ou boca provocar a contaminação de tais profissionais (CDC, 2021).

Para evitar a contaminação, enquanto a vacina não estava disponível, os cirurgiões-dentistas foram orientados a realizarem atendimentos restritos às urgências e aconselhados a promoverem triagens através de consultas remotas. As consultas presenciais, recomendadas a um grupo de pacientes, selecionados após apropriada avaliação de risco, devem seguir protocolos globais de proteção individual para o atendimento clínico durante a COVID-19 e um tempo entre as consultas deve ser reservado visando a excelente higienização e descontaminação de instrumentos, equipamentos e ambiente, assim como afastamento dos pacientes (COULTHARD, 2020; NHS, 2021).

Sendo o Tratamento Restaurador Atraumático (TRA) considerado como uma abordagem minimamente invasiva, podendo ser utilizado em abordagens preventivas, terapêuticas e restauradoras (NAVARRO et al., 2015; MONNERAT, 2015), tornando-o compatível com estratégias de atuação odontológica no enfrentamento à COVID-19, esta revisão integrativa verificou na literatura as 
indicações do TRA, com o objetivo de responder à pergunta "Por que o TRA foi indicado na pandemia do SARS Cov-2?".

\section{MATERIAIS E MÉTODOS}

Revisão integrativa, que delimitou as seguintes etapas percorridas: 1) identificação da temática (elaboração da pergunta norteadora, estabelecimento de descritores e dos critérios de inclusão/exclusão de artigos); 2) amostragem (seleção dos artigos); 3) categorização dos estudos; 4) definição das informações a serem extraídas dos trabalhos revisados; 5) análise e discussão a respeito das indicações; 6) síntese do conhecimento evidenciado nos artigos analisados e apresentação da revisão integrativa.

Estabeleceu-se a seguinte pergunta norteadora: "Por que o TRA foi indicado na pandemia do SARS Cov-2?". Os critérios de inclusão adotados pelo presente estudo foram: a publicação ter como temática o tratamento restaurador atraumático e o SARS-CoV-2 ou Covid-19; publicações classificadas como artigo original e revisões bibliográficas, com, no máximo, 5 anos de publicação, divulgadas em língua portuguesa, espanhola ou inglesa; publicações completas com resumos disponíveis e indexados nas bases de dados: PubMed, Lilacs e Scielo. Foram excluídos os editoriais, as cartas ao editor, os estudos reflexivos, bem como estudos que não abordassem a temática relevante ao objetivo da revisão.

A pesquisa nas bases de dados foi executada de março a setembro de 2021, através de terminologias em saúde consultadas nos Descritores em Ciências da Saúde (DECS) e no Medical Subject Headings (MeSH), pelos quais se identificaram os respectivos descritores: tratamento restaurador atraumático (atraumatic restoratrive treatment), COVID-19, SARS Cov-2, aerossol (aerosol) e biossegurança (biosecurity).

As referências foram analisadas mediante um formulário adaptado de Ursi. Este possibilitou a análise em relação aos seguintes aspectos: identificação do estudo (título do artigo, autores, país, idioma, ano de publicação); revista científica; e características metodológicas do estudo (tipo de publicação, objetivo, amostra, tratamento dos dados, resultados, implicações, nível de 
evidência). Os artigos foram organizados por ano de publicação e classificados por níveis de evidência ( $\mathrm{a}$ VI, I = maior), segundo Stetler e colaboradores (STETLER et al.,1998).

A seguir foram extraídos os principais dados com o uso do instrumento supracitado. O objetivo dessa etapa foi organizar e resumir as informações que relacionam o tratamento restaurador atraumático como uma indicação durante tratamentos odontológicos, na pandemia do SARS-CoV-2. As informações abrangeram a amostra do estudo, os objetivos, a metodologia empregada, os resultados e as principais conclusões de cada estudo.

Assim, após percorridos os trâmites metodológicos descritos, foram selecionados os artigos que contemplavam a pergunta norteadora do presente trabalho, bem como os que atenderam aos critérios previamente estabelecidos. Os aspectos éticos relativos à realização de pesquisas científica foram respeitados. A análise das indicações do tratamento restaurador atraumático ocorreu mediante avaliação da metodologia de cada artigo e observações teóricas ou práticas feitas pelos autores.

\section{RESULTADOS}

Foram localizadas 84 publicações, distribuídas nas bases de dados utilizadas nas buscas realizadas. Deste total, foram excluídos 30 artigos científicos pelo fato de não se apresentarem pertinentes com o presente estudo. Sendo assim, nesta revisão integrativa foram classificados como potencialmente relevantes um total de 54 estudos, resultantes da leitura e análises de seus títulos e resumos; no entanto, 45 estudos não apresentavam informações para colaborar com o questionamento desta pesquisa e, finalmente, foram utilizados 9 artigos científicos.

De acordo com o tipo de delineamento dos artigos científicos analisados, observou-se que o tipo de estudo predominante foi a revisão integrativa e o país com mais publicações foi o Brasil. Os artigos selecionados compreendem 
publicações nos anos de 2020 e 2021, sendo apresentados na tabela 1. Tais resultados desta revisão integrativa são relatados a seguir.

Segundo Franco, de Camargo e Peres, na revisão de literatura intitulada "Cuidados odontológicos na era da COVID-19: recomendações para procedimentos odontológicos e profissionais", publicado em 2020, no Brasil, o TRA é indicado para a proteção dos pacientes, equipes auxiliares e dos cirurgiões-dentistas, na era da COVID-19 (FRANCO; DE CAMARGO; PERES, 2020).

O grupo de pesquisadores chineses, composto por GE e colaboradores, publicou uma revisão integrativa com o título "Possible aerosol transmission of COVID-19 and special precautions in dentistry", no ano de 2020, onde relataram que o TRA é indicado devido à análise de transmissão de aerossol e gotículas, no ambiente odontológico (GE et al., 2020).

Rocha e colaboradores, através de uma pesquisa realizada no Brasil, publicaram, em 2020, uma revisão bibliográfica qualitativa com o título "Odontologia no contexto da pandemia por COVID-19: uma visão crítica", onde apontaram a indicação do TRA para evitar o uso de periféricos de rotação e todos que geram aerossóis (ROCHA et al., 2020).

Realizando uma revisão integrativa, em 2020, no Brasil, Medeiros e colaboradores destacaram que a produção de aerossóis é o agente de maior periculosidade, durante os procedimentos odontológicos, cabendo ao dentista dar preferência às técnicas restauradoras atraumáticas (MEDEIROS et al., 2020).

Um grupo polonês, publicou um artigo científico com o título "The impact of the COVID-19 pandemic on the spectrum of performed dental procedures", em 2021, onde indicaram a técnica restauradora atraumática para a redução do aerossol e como método não invasivo ou minimamente invasivo (NIJAKOWSKI et al., 2021).

Machado e colaboradores, em 2020, no Brasil, publicaram uma revisão integrativa com o título "Biossegurança e retorno das atividades em Odontologia: aspectos relevantes para enfrentamento de COVID-19", na qual mencionaram 
que, devido a preocupação na produção de aerossóis, instrumentos ultrassônicos devem ser trocados, neste período, por instrumentos manuais, dando preferência aos procedimentos restauradores atraumáticos (MACHADO et al., 2020).

Com o título "Guía para el trabajo clínico em Odontología durante pandemia por SARS-CoV-2, em el Hospital Clínico Dra. Eloísa Díaz", Miranda, Hiza e Yañez, publicaram, no Chile, em 2021, uma revisão de literatura mencionando que em tratamentos onde não seja possível isolar, preferir técnicas como o TRA ou instrumentação manual (MIRANDA; HIZA; YAÑEZ, 2021).

Barbosa e colaboradores, no Brasil, em 2021, publicaram um relato de caso clínico com o título "Manejo da cárie dentária e comportamento infantil durante a pandemia de COVID-19: relato de caso", onde o tratamento restaurador atraumático foi indicado para reduzir o risco de contaminação cruzada e proporcionar um atendimento mais confortável e uma abordagem mais conservadora nos tratamentos infantis (BARBOSA et al., 2021). O TRA foi recomendado como uma das medidas especiais de segurança, na revisão integrativa, realizada no Líbano, em 2021, por Elzein e colaboradores, com o título "Legal liability facing COVID-19 in dentistry: Between malpractice and preventive recommendations" (ELZEIN et al., 2021). 
O tratamento restaurador atraumático e a pandemia do SARS-COV-2

Reis LMS, Paula JA, Silva GA, Valladão ASN

Tabela 1 - Resultados obtidos através da utilização do instrumento adaptado de Ursi (URSI, 2005). 


\begin{tabular}{|c|c|c|c|c|}
\hline Título & $\begin{array}{c}\text { Desenho do } \\
\text { Estudo }\end{array}$ & Ano & País & Por que indicaram o TRA? \\
\hline $\begin{array}{l}\text { Cuidados odontológicos } \\
\text { na era da COVID-19: } \\
\text { recomendações para } \\
\text { procedimentos } \\
\text { odontológicos e } \\
\text { profissionais. }\end{array}$ & $\begin{array}{c}\text { Revisão } \\
\text { bibliográfica }\end{array}$ & 2020 & Brasil & $\begin{array}{l}\text { Para a proteção dos pacientes; } \\
\text { equipes auxiliares e os cirurgiőes- } \\
\text { dentistas na era da COVID-19. }\end{array}$ \\
\hline $\begin{array}{c}\text { Possible aerosol } \\
\text { transmission of COVID-19 } \\
\text { and special precautions in } \\
\text { dentistry. }\end{array}$ & $\begin{array}{l}\text { Revisão } \\
\text { integrativa }\end{array}$ & 2020 & China & $\begin{array}{l}\text { Devido à análise de transmissão pelo } \\
\text { aerossol e gotículas no ambiente } \\
\text { odontológico. }\end{array}$ \\
\hline $\begin{array}{l}\text { Odontologia no contexto } \\
\text { da pandemia por COVID- } \\
\text { 19: uma revisão crítica. }\end{array}$ & $\begin{array}{c}\text { Revisão } \\
\text { bibliográfica. }\end{array}$ & 2020 & Brasil & $\begin{array}{l}\text { Para evitar instrumentos de rotação } \\
\text { e todos que gerassem aerossóis. }\end{array}$ \\
\hline $\begin{array}{l}\text { COVID-19 pandemic } \\
\text { impacts to dentistry. }\end{array}$ & $\begin{array}{l}\text { Revisão } \\
\text { integrativa }\end{array}$ & 2020 & Brasil & $\begin{array}{l}\text { Destacaram a produção de aerossóis } \\
\text { como agente de grande } \\
\text { periculosidade durante os } \\
\text { procedimentos odontológicos, } \\
\text { indicando o TRA. }\end{array}$ \\
\hline $\begin{array}{l}\text { The impact of the COVID- } \\
19 \text { pandemic on the } \\
\text { spectrum of performed } \\
\text { dental procedures. }\end{array}$ & $\begin{array}{l}\text { Estudo } \\
\text { analítico }\end{array}$ & 2021 & Polônia & $\begin{array}{l}\text { Para reduzir a geração de aerossol } \\
\text { em procedimentos e usar métodos } \\
\text { não invasivos e minimamente } \\
\text { invasivos, indicando o TRA. }\end{array}$ \\
\hline $\begin{array}{l}\text { Biossegurança e retorno } \\
\text { das atividades em } \\
\text { odontologia: aspectos } \\
\text { relevantes para } \\
\text { enfrentamento de COVID- } \\
19 .\end{array}$ & $\begin{array}{l}\text { Revisão } \\
\text { integrativa }\end{array}$ & 2020 & Brasil & $\begin{array}{c}\text { Devido a preocupação na produção } \\
\text { de aerossóis, indicando o TRA }\end{array}$ \\
\hline $\begin{array}{l}\text { Guia para el trabajo } \\
\text { clínico em odontología } \\
\text { durante pandemia por } \\
\text { Sars-Cov-2, em el Hospital } \\
\text { Clínico Dra. Eloísa Díaz. }\end{array}$ & $\begin{array}{l}\text { Revisão } \\
\text { bibliográfica }\end{array}$ & 2021 & Chile & $\begin{array}{l}\text { Para prevenir a transmissão e } \\
\text { diminuir infecções. }\end{array}$ \\
\hline $\begin{array}{l}\text { Manejo da cárie dentária } \\
\text { e comportamento infantil } \\
\text { durante a pandemia de } \\
\text { covID-19: Relato de caso. }\end{array}$ & $\begin{array}{l}\text { Relato de } \\
\text { caso }\end{array}$ & 2021 & Brasil & $\begin{array}{l}\text { Reduzir o risco de contaminação } \\
\text { cruzada e proporcionar um } \\
\text { atendimento mais confortável para a } \\
\text { criança. Permitir uma abordagem } \\
\text { conservadora. }\end{array}$ \\
\hline $\begin{array}{l}\text { Legal liability facing } \\
\text { COVID-19 in dentistry: } \\
\text { Between malpractice and } \\
\text { preventive } \\
\text { recommendations. }\end{array}$ & $\begin{array}{l}\text { Revisão } \\
\text { integrativa }\end{array}$ & 2021 & Líbano & $\begin{array}{l}\text { Possível solução para o dilema da } \\
\text { transmissão do SARS-CoV-2, } \\
\text { indicando o TRA como uma das } \\
\text { medidas especiais de segurança. }\end{array}$ \\
\hline
\end{tabular}

\section{DISCUSSÃO}


Os estudos analisados mostraram que o TRA foi indicado para tratamentos odontológicos durante a pandemia do SARS-CoV-2. Todos os estudos relacionaram, de alguma forma, a indicação do TRA à redução da produção de aerossol (FRANCO; DE CAMARGO; PERES, 2020; GE et al., 2020; ROCHA et al., 2020; MEDEIROS et al., 2020; NIJAKOWSKI et al., 2021; MACHADO et al., 2020; MIRANDA; HIZA; YAÑEZ, 2021; BARBOSA et al., 2021; ELZEIN et al., 2021), pelo fato da técnica utilizar, geralmente, instrumentos manuais para a abordagem de acesso à cavidade e remoção do tecido cariado (MOLINA; FAULKS; FRENCKEN, 2015). Esta preocupação corrobora com algumas pesquisas que especulam o fato do espalhamento de gotículas ou aerossóis, gerados a partir de expirações violentas ou não-violentas de vírus infectados com o SARS-CoV-2, serem responsáveis pelo maior papel na transmissão aérea da doença COVID-19. Tal espalhamento do vírus ainda pode ter a influência de diversos fatores como temperatura, humidade e ventilação, tornando-o mais grave (MORAWSKA, 2006; TELLIER, 2009; JUDSON; MUNSTER, 2019; MORAWSKA; CAO, 2020; WANG; DU, 2020; JAYAWEERA et al., 2020).

Adicionalmente, no contexto da COVID-19, apesar de não apresentarem a unanimidade da indicação da redução do aerossol, a técnica restauradora atraumática também foi citada como alternativa para proporcionar conforto e tratamentos mais conservadores em crianças (BARBOSA et al., 2021); proteção de pacientes, equipes auxiliares e cirurgiões-dentistas (FRANCO; DE CAMARGO; PEREZ, 2020); prevenir a transmissão e diminuir infecções (MIRANDA; HIZA; YAÑEZ, 2021) e como uma medida especial de segurança contra a transmissão do SARS-CoV-2 (ELZEIN et al., 2021).

O custo efetivo do TRA, em relação a outros métodos de restauração, não foi citado como uma característica para indicação no contexto pandêmico do SARS-CoV-2, nos trabalhos incluídos nesta pesquisa. No entanto, Tonmukayakul e Arrow, em 2017, citam esta característica do TRA, sendo considerada crucial, uma vez que a pandemia impactou economicamente a população mundial (TONMUKAYAKUL; ARROW, 2017; NICOLA et al., 2020). 
Apesar de Barbosa e colaboradores, em 2021, terem mencionado a indicação do uso do TRA, no momento da pandemia, para o tratamento de crianças, não foi observada a indicação, nos trabalhos pesquisados, para 0 tratamento de adultos ou idosos. Neste sentido, da Mata e colaboradores, em 2019, ressaltam a preferência de pacientes idosos pelo TRA, uma vez que tal tratamento, na maioria das vezes, não requer o uso de anestesia ou brocas em alta ou baixa rotação. Além desta vantagem, que minimiza o estresse adicional vivido por tais pacientes durante a COVID-19, um ponto estratégico do TRA é a possibilidade do cirurgião-dentista prestar atendimento fora do ambiente clínico tradicional, ou seja, no domicílio do paciente ou em abrigos de idosos (BARBOSA et al., 2021; DA MATA et al., 2019).

\section{CONCLUSÃO}

Considerando um momento com tamanha adversidade, como o vivido durante a pandemia do SARS-CoV-2, colaborar com práticas odontológicas baseadas em evidências científicas agrega informação para o atendimento da população, gerando maior conforto e segurança à equipe envolvida. Neste contexto, a presente revisão integrativa mostra que o TRA está indicado para o tratamento de pacientes, nas diversas faixas etárias, sobretudo por evitar a propagação do aerossol.

\section{REFERÊNCIAS}

ALI, K., RAJA, M. Coronavirus disease 2019 (COVID-19): challenges and management of aerosol-generating procedures in dentistry. Evidence Based Dentistry, n.21, p.44-45, 2020.

BARBOSA, M.G., SILVA, N.R., OLIVEIRA, S.S.B. et al. Manejo da cárie dentária e comportamento infantil durante a pandemia de COVID-19: relato de caso. Rev Odontol Bras Central, n.30, p.209-21, 2021.

CDC - CENTERS FOR DISEASE CONTROL AND PREVENTION. Interim guidance for managing healthcare personnel with SARS-CoV-2 infection or exposure to SARS-CoV-2. Disponível em https://www.cdc.gov/coronavirus/2019ncov/hcp/guidance-risk-assesment-hcp.html (acessado em setembro/2021). 
COULTHARD, P. The oral surgery response to coronavirus disease (COVID-19). Keep calm and carry on? Oral Surg, n.13, p.95-7, 2020.

DA MATA, C., MCKENNA, G., ANWEIGI, L. et al. Na RCT of atraumatic restorative treatment for older adults: 5 year results. J Dent, n.83, p.95-9, 2019.

ELZEIN, R., BADER, B., RAMMAL, A. et al. Legal liability facing COVID-19 in dentistry: Between malpractice and preventive recommendations. Journal of Forensic and Legal Medicine, n.78, p.102123, 2021.

FRANCO, J.B., DE CAMARGO, A.R., PERES, M.P.S.M. Cuidados odontológicos na era do COVID-19: recomendações para procedimentos odontológicos e profissionais. Rev Assoc Paul Cir Dent, n.74, p.18-21, 2020.

GE, Z-Y., YANG, L-M., XIA, J-J. et al. Possible aerosol transmission of COVID19 and special precautions in dentistry. J Zhejiang Univ-Sci B, n.21, p.361-8, 2020.

JAYAWEERA, M., PERERA, H., GUNAWARDANA, B. et al. Transmission of COVID-19 virus by droplets and aerosols: A critical review on the unresolved dichotomy. Environmental Research, n.188, p.109819, 2020.

JUDSON, S.D., MUNSTER, V.J. Nosocomial transmission of emerging viruses via aerosol-generating medical procedures. Viruses, n.11, p.940, 2019.

KANDEEL, M., AL-NAZAWI, M. Virtual screening, and repurposing of FDA approved drugs against COVID-19 main protease. Life Sci, n.251, p.117627, 2020.

MACHADO, G.M., KASPER, R.H., BUSATO, A.L.S. et al. Biossegurança e retorno das atividades em Odontologia: aspectos relevantes para enfrentamento de COVID-19. Stomatos, n.50, p.30-45, 2020.

MEDEIROS, M.S., SANTOS, H.L.F., BARRETO, J.O. et al. COVID-19 pandemic impacts to Dentistry. RGO, Rev Gaúch Odontol, n.68, p.e20200021, 2020.

MIRANDA, I.A., HIZA, C.R., YAÑEZ, Y.M. Guía para el trabajo clínico em Odontología durante pandemia por SARS-CoV-2, em el Hospital Clínico Dra. Eloísa Díaz. Int J Odontostomat, n.15, p.51-8, 2021.

MOLINA, G.F., FAULKS, D., FRENCKEN, J. Acceptability, feasibility and perceived satisfaction of the use of the atraumatic restorative treatment approach for people with disability. Braz Oral Res [online], n.29, p.1-9, 2015.

MORAWSKA, L. Droplet fate in indoor environments, or can we prevent the spread of infection? Indoor Air, n.16, p.335-47, 2006.

MORAWSKA, L., CAO, J. Airborne transmission of SARS-CoV-2: the world should face the reality. Environ Int, n.139, p.105730, 2020. 
NAVARRO, M.F.L., LEAL, S.C., MOLINA, G.F. et al. Tratamento restaurador atraumático: atualidades e perspectivas. Rev Assoc Paul Cir Dent, n.69, p.289301, 2015.

NHS. Guidance - COVID-19: infection prevention and control dental appendix. Disponível em: https://www.gov.uk/government/publications/wuhan-novelcoronavirus-infection-prevention-and-control/covid-19-infection-prevention-andcontrol-dental-appendix (acessado em setembro/2021).

NICOLA, M., ALSAFI, Z., SOHRABI, C. et al. The socio-economic implications of the coronavirus pandemic (COVID-19): A review. Int J Surg, n.78, p.185-193, 2020.

NIJAKOWSKI, K., CIESLIK, K., LAGANOWSKI, K. et al. The impact of the COVID-19 pandemic on the spectrum of performed dental procedures. Int $\mathbf{J}$ Environ Res Public Health, n.18, p.3421, 2021.

MONNERAT, A.F. Tratamento restaurador atraumático: abordagem clínica em saúde pública. 1ํㅡㄹ ed. Rio de Janeiro: Elsevier, 2015. p.1-10.

ROCHA, J.R., NEVES, M.J., GUILHERME, H.G. et al. Odontologia no contexto da pandemia por COVID-19: uma visão crítica. Braz J Hea Rev, n.6, p.19498509, 2020.

STETLER, C.B., MORSI, D., RUCKI, S. et al. Utilization-focused integrative reviews in a nursing service. Appl Nurs Res, n.11, p.195-206, 1998.

TELLIER, R. Aerosol transmission of influenza A virus: a review of new studies. J R Soc Interface, n.6, p.S783-90, 2009.

TONMUKAYAKUL, U., ARROW, P. Cost-effectiveness analysis of the atraumatic restorative treatment-based approach to managing early childhood caries. Community Dent Oral Epidemiol, n.45, p.92-100, 2017.

URSI, E.S. Prevenção de lesões de pele no perioperatório: revisão integrativa da literatura. [dissertação]. Ribeirão Preto: Universidade de São Paulo, Escola de Enfermagem de Ribeirão Preto; 2005.

WANG, J., DU, G. COVID-19 may transmit through aerosol. Ir J Med Sci, n.189, p.1143-4, 2020. 